\title{
A park with a kangaroo problem
}

\author{
David Cheal
}

Hattah-Kulkyne National Park in Victoria, Australia, is a large reserve important for its vegetation communities, These were once extensive in the region, but are now reduced to remnants due to clearance for agriculture. The vegetation in the Park has suffered from overgrazing, and the rehabilitation programme includes culling kangaroos. Population management of this kind often generates controversy, and the author, a research botanist, explains why it is necessary in this Park.

Hattah-Kulkyne National Park, established in stages between 1969 and 1980 , is a large $(48,000$ ha) biosphere reserve in the semi-arid north-west of the state of Victoria, Australia (Figure 1). As much of the surrounding land has been alienated and cleared for agriculture, it is an important remnant and habitat island for formerly extensive vegetation communities and their dependent fauna. Because of extreme habitat degradation resulting from timber-cutting and grazing by livestock in the past and from present grazing by kangaroos and rabbits, an integrated habitat rehabilitation programme was implemented. This programme required the culling of kangaroos from part of the Park. The reasons for the cull and the background to the Park's rehabilitation programme are summarized below.

\section{Change in the fauna}

The first collecting expeditions to the north-west of Victoria in 1856/57 collected 30 native mammal species. Thirteen of these have not been recorded in Victoria since the nineteenth century, including species originally described as "very Park with a kangaroo problem plentiful'! In 1856/57 the large kangaroos were described in the first collecting expedition's diaries as 'very rare' or 'scarce' (Wakefield, 1966). However, following European settlement of the area, the larger kangaroos markedly increased in abundance within Hattah-Kulkyne (the major national park of the region) to the extent that they now exert a new and heavy grazing pressure on the native vegetation communities.

There are six species of kangaroo in Australia, and none is considered to be at risk. Indeed, in some areas they appear to have increased following developments associated with the provision of water and pasture improvement (Poole, 1979). Two kangaroo species occur in the Park. The red kangaroo Macropus rufus prefers the grassy plains of the interior of Australia and is uncommon in the riverine woodlands and dwarf eucalypt shrublands (mallee) of Hattah-Kulkyne. This species is low in numbers in the Park and is

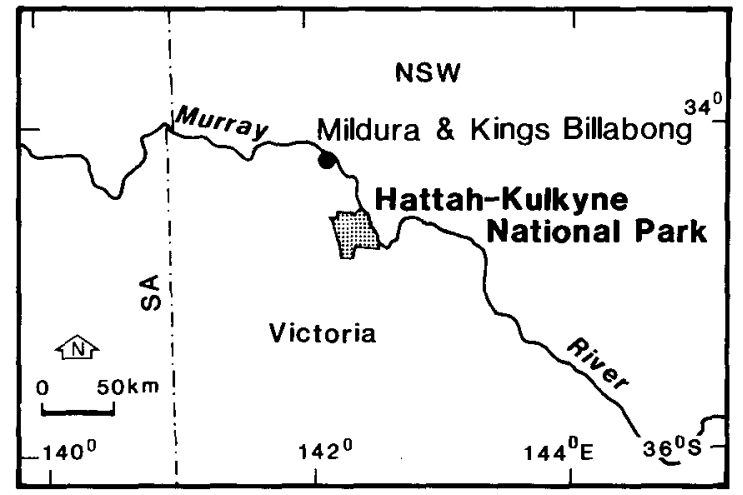

Figure 1. Location of Hattah-Kulkyne National Park 
not subject to population management. By contrast, the western grey kangaroo Macropus fuliginosus, more characteristic of woodlands and shrublands, is now abundant. Recent surveys in the Park (1982) calculated the density of western grey kangaroos at $45 \pm 6$ per sq $\mathrm{km}$ (i.e. $21,500 \pm 2700$ for the Park). The dramatic drought of 1982/83 led to a substantial decline in kangaroo numbers, with mortality estimated at 42 per cent. Nevertheless, even after this population decline, density remained 'high' [i.e. greater than 10 per sq km (sensu Caughley and Grigg, 1982)].Western grey kangaroos are widespread across southern Australia, and in Victoria are recorded from at least four national parks and seven state parks or other fauna reserves, which together cover an area of 545,480 ha.

\section{Early alienation}

Early grazing runs established after 1847 included areas that are now part of the Park. Stocking rates for sheep and cattle were high, and the then dense stands of cypress-pine Callitris spp. and she-oak Casuarina spp. were ring-barked and burnt in order to open up the country for stock grazing. The grasses and other herbs were thus promoted at the expense of the shrubs and trees. Logging of cypress-pine continued until the early 1940 s. Since 1878, rabbits Oryctolagus cuniculus have become widespread, frequently attaining very high densities.

Thus, from late last century, Hattah-Kulkyne has been extensively overgrazed. Grazing by sheep was stopped in 1963, but some grazing by cattle continued until 1977. Unfortunately, the removal of domestic stock did not lead to vegetation regeneration. Rabbit control, except for small areas of less than 50 ha, has been ineffective, and the replacement of cypress-pine/she-oak woodlands by broad swathes of grassland has produced highly suitable habitat for the larger kangaroos.

\section{Predation}

Apart from feed availability, hunting by aborigines and predation by dingoes Canis familiaris dingo also limited kangaroo numbers in the past. Aborigines have not exerted any signi96 ficant hunting pressure since last century when their local populations were decimated. Shepherd (1981) provided evidence of dingoes limiting kangaroo numbers in the absence of human interference in dingo populations, and evidence of a dramatic increase in kangaroo numbers following dingo reduction. Even as early as 1857 , Krefft recorded that, 'The dingo is not so plentiful as in former years as the strychnine of the settler has killed a great many, and only a very few individuals are to be met with ...' (Wakefield, 1966). Dingo reduction and elimination rapidly followed settlement and the introduction of grazing stock.

Consequently, the development of more suitable habitat and the removal of predation have led to a dramatic increase in western grey kangaroo numbers. As red kangaroos are at very low density throughout the Park, and are thus not subject to population management, they are not considered further in this article, and all subsequent reference to kangaroos is solely concerned with western grey kangaroos.

\section{Vegetation regeneration}

For the kangaroos, late summer to autumn is a period when food is normally critically short, as at this time the annual forage has disappeared and growth of perennial herbs is much reduced by seasonal drought. The intense grazing pressure formerly exerted by high numbers of domestic stock and currently exerted by kangaroos and rabbits at this time has led to the near elimination of perennial herbs and their replacement by winter-green annuals, mostly weeds. As a result, there is a severe impact on the few perennial herbs that remain and on the seedling and sucker regeneration of the remnant shrubs and trees. Most of the Park is still experiencing severe habitat destruction and vegetation degradation.

Immediately following the declaration of the Hattah-Kulkyne National Park in April 1980, the National Parks Service (Victoria) started a rehabilitation programme over a 5700 ha section of the Park, known as the Mournpall Paddock. This large area was fenced to exciude rabbits, which were then progressively greatly reduced in numbers inside. Although the rabbit reduction has been remarkably successful, vegetation

Oryx Vol 20 No 2. April 1986 
regeneration, which was notable in 1981 within the treated area, did not continue in 1982 or beyond. By 1984 there was no discernible difference between the Mournpall Paddock (effectively rabbit-free) and the rest of the Park. As part of the rehabilitation programme, a coordinated vegetation monitoring programme was instituted in 1980. Small exclusion plots (of 100 sq $\mathrm{m})$ were erected to exclude rabbits and kangaroos. Annual monitoring of these and other plots has described the dramatic vegetation changes following release from grazing. Not surprisingly, there is significantly greater cover within the exclosures than on adjacent controls, but there are also significant qualitative differences between the exclosures and the controls. Outside, the open-herbland is dominated by winter-green annuals in most years, of exotic genera such as Brassica, Medicago, Pentaschistis, and Schismus. Particularly benign seasons may favour the perennial grasses, but these are rare events. Usually the weeds dominate. Inside the plots, perennial tussock grasses, largely Stipa spp., now dominate and there is increased species richness, including native perennial herbs, such as the legumes Psoralea and Swainsona, which are now relatively rare outside. There is also some regeneration of formerly dominant trees and shrubs, such as Acacia, Callitris, Dodonaea and Hakea, where these remnant woody plants occur near the exclosure plots. Outside these small exclosures such regeneration is virtually non-existent.

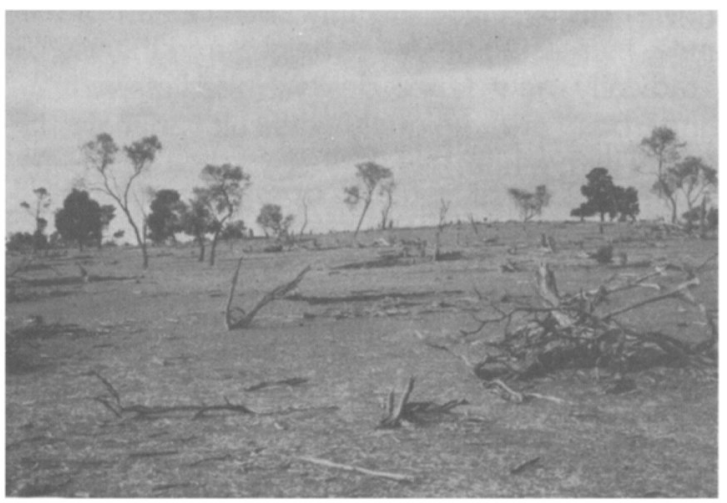

Degraded woodland with remnant Callitris preissii (Cupressaceae) and Hakea tephrosperma (Proteaceae) (David Cheal)

Park with a kangaroo problem

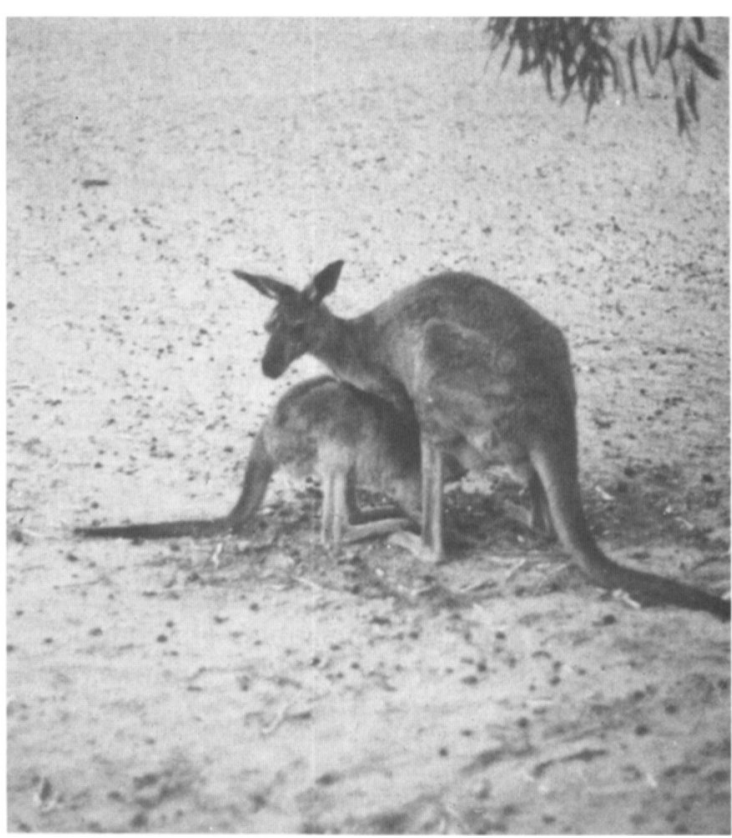

Western grey kangaroos. Hattah-Kulkyne National Park, February 1983. Note the lack of herbaceous cover and abundance of kangaroo faeces (A. Mitchell).

Once the grazing pressure has been reduced, it is expected that spontaneous vegetation regeneration will be sufficient to restore near-original vegetation communities to most of the Park's (former) woodlands. However, over broad areas even the last few trees and shrubs have been eliminated and there is no longer a local seed source. In such situations the woody species may need to be reintroduced by planting young seedlings grown from locally collected seed. Where vegetation degradation has been so severe that mobile dunes have developed and spread, soil conservation works have proved necessary.

\section{Grazing effects}

Approximately 30 per cent of the National Park once supported extensive woodland (as defined by Specht, 1972), dominated by cypress-pine and she-oaks. With a few exceptions, all woody plants of this major vegetation community are killed by wildfires, which occasionally occur in this summer-dry climate. Nowadays, fires are almost completely restricted to the mallee (dense dwarf 
eucalypt shrublands) as the available fuel elsewhere is now too discontinuous to carry a fire. In the non-riverine woodlands, regeneration after fires, logging or other (past) disturbance is solely or largely from seed. For many decades such re-establishment has not occurred because those seedlings that do germinate are removed by grazing. Instead, areas that once supported cypress-pine/she-oak woodland now support open herbland, sometimes with an occasional veteran of one of the formerly abundant trees or shrubs. Even where stock no longer graze and rabbit grazing pressure is now negligible, as in the Moumpall Paddock, there is still no regeneration. Although the preferred diet is grasses, kangaroos will remove the regenerant shrubs and seedlings when there is little else available. This happens nearly every year at the end of summer and through autumn.

Fourteen plant species endangered in Victoria are known to occur in Hattah-Kulkyne National Park. The survival of at least seven of these is threatened by grazing (pers. obs., plus Parsons et al. 1985). Comparison with similar areas nearby with significantly lower grazing pressure (such as Kings Billabong) has shown that these rare species, all of which are perennial, summer-green herbs, grow, reproduce and regenerate successfully when their habitats are not subject to the degradation attributable to overgrazing.

Such massive habitat disruption has also, not surprisingly, affected the dependent fauna. The remaining mammals seem relatively secure, but the same cannot be said for the other vertebrates. The last spotted bowerbird Chlamydera maculata seen in Victoria was recorded from the area in 1975. The dense thickets required by this species, and many of its food plants, have been eliminated from the Park. Similarly, other birds once common are now rare, notably bush thick-knee Burhinus magnirostris, pink cockatoo Cacatua leadbeateri and white-browed treecreeper Climacteris affinis.

The removal of stock from the Park and the great reduction of rabbits within the Mournpall Paddock reduced the variety of grazing animals, but did not significantly reduce the grazing pressure. 98

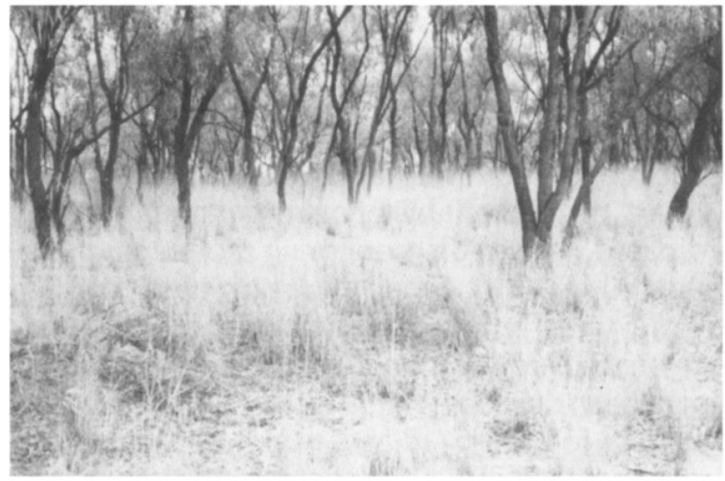

Riverain woodland. Kings Billabong, June 1983. There is significantly lower grazing pressure here than in HattahKulkyne National Park (David Cheal)

The western grey kangaroo has replaced these animals as the principal grazer. Consequently, the necessary reduction in grazing pressure to permit vegetation regeneration involves reduction in the numbers of kangaroos.

\section{Habitat restoration}

Kangaroo population reduction was restricted to the Mournpall Paddock (12 per cent of the Park area), since elsewhere rabbits were causing at least as much damage as kangaroos, and reduction of kangaroo numbers would have provided extra forage and thus led to a great increase in rabbit numbers. In order to prevent kangaroos re-entering the Mournpall Paddock after population reduction, the fencing erected as part of the rabbit control has been upgraded to be kangarooproof. This involved electrification of part of the fence, increasing the fence height, and the use of a radically new fence design (needless to say kangaroos are uniquely difficult animals to control with fences).

After a meeting with conservation and certain animal welfare groups in 1983, it was decided to attempt to muster excess kangaroos out of the Mournpall Paddock, even though it was recognized that this would merely be moving the overgrazing problem to elsewhere in the Park. From 3 to 5 July 1984, 16 horsemen supervised by two Park staff attempted to slowly drive kangaroos out of each of four sectors of the Paddock. Unfortunately, the muster proved unsuccessful. It was extremely stressful to the kangaroos, leading to a

Oryx Vol 20 No 2, April 1986 


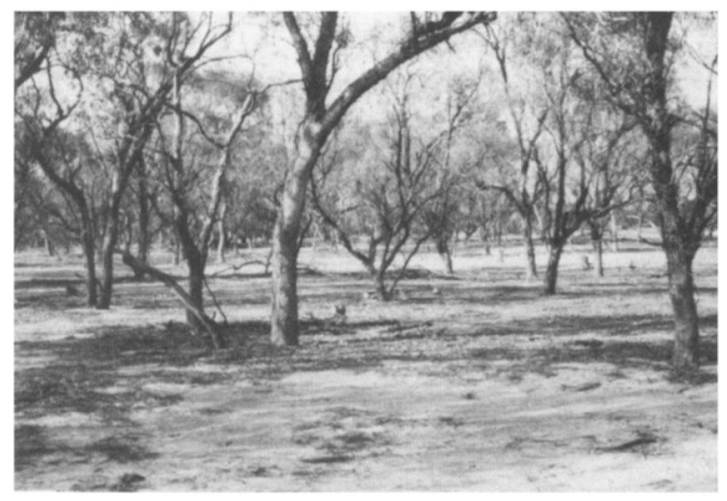

Riverain woodland. Hattah-Kulkyne National Park, June 1983 (David Cheal).

number of inadvertant deaths, and was ineffective in achieving the necessary population reduction inside the Paddock. Culling remained the only practicable humane method to reduce the grazing pressure. In August 1984, 787 kangaroos in the Moumpall Paddock were shot by carefully supervised professional shooters and the carcasses buried. In July 1984, the total population for the Mournpall Paddock was estimated as 1620 western grey kangaroos. Thus, their estimated density after the cull is about 14.6 per sq $\mathrm{km}$. There is no attempt to eliminate western grey kangaroos from the Park or from the Mournpall Paddock-merely to reduce their numbers to a level that would permit vegetation regeneration and survival of its dependent biota. The carcasses and skins do not leave the Park and thus do not enter the commercial kangaroo industry (kangaroos are not killed for commercial purposes in Victoria).

The population reduction, and occasional culling to keep the kangaroo numbers in the Mournpall Paddock low, is aimed solely at reducing overgrazing in the Park. The most recent (July 1985) population census estimates are 14,210 $( \pm 1740)$ western grey kangaroos for the Kulkyne Parks and $380( \pm 210)$ red kangaroos, i.e. a total kangaroo density of 29.6 ( \pm 3.9 ) kangaroos per sq $\mathrm{km}$. The comparable figures for the Moumpall Paddock alone are $1130( \pm 120)$ western grey kangaroos, approximately 5 red kangaroos and a total kangaroo density of 19.8 ( \pm 2.1 ) per sq km. Vegetation regeneration will take many years as Park with a kangaroo problem growth is slow in this semi-arid climate (mean annual rainfall approximately $340 \mathrm{~mm}$ ). Once habitat is restored in the Mournpall Paddock, then the rehabilitation programme can be extended to other sections of the Park. In future it may be possible to reintroduce those species that have disappeared from the region. At the very least, re-establishment of the woodlands, with a consequent decrease in the herbaceous cover, may provide some natural control over kangaroo populations and the catastrophic declines that occur during the occasional droughts. Regenerated woodlands could once again provide secure habitat for many plants and animals now threatened outside reserves. Until overgrazing is reduced their survival inside reserves is also threatened.

\section{Acknowledgments}

Professor A. K. Lee and Dr R. F. Parsons critically reviewed this manuscript, and their valuable suggestions and on-going assistance is gratefully acknowledged. This paper is published with the approval of the Director of Fisheries and Wildlife, Victoria, and the Director of the National Parks Service, Victoria. Kangaroo censuses were conducted by teams from the Australian National Parks and Wildlife Service, and by $\mathrm{Mr}$ D. Morgan of the Melbourne College of Advanced Education. Park rehabilitation could not have proceeded without the dedicated efforts of National Park Service staff, particularly in fencing, vermin control and kangaroo management.

\section{References}

Caughley, G. and Grigg, G.C. 1982. Numbers and distribution of kangaroos in the Queensland pastoral zone. Aust. Wildl. Res. 9, 365-371.

Parsons, R.F., Scarlett, N.H. et al. 1985 (on going). A Register of Rare and Endangered Native Plants in Victoria. Department of Botany, La Trobe University, Bundoora, Victoria.

Poole, W.E. 1979. The status of the Australian Macropodidae. In The Status of Endangered Australasian Wildlife: Proceedings of the Centenary Symposium of the Royal Zoological Society of South Australia, Adelaide (ed. H.J. Tyler).

Shepherd, N.C. 1981. Predation of red kangaroo, Macropus rufus, by the dingo Canis familiaris dingo (Blumenbach), in north-western New South Wales. Aust. Wildl. Res. 8, $255-262$

Specht, R.L. 1972. The Vegetation of South Australia. Government Printer, Adelaide, South Australia

Wakefield, N.A. 1966. Mammals of the Blandowski Expedition to north-western Victoria. Proc. R. Soc. Vict. 79, $371-391$

David Cheal, National Parks Service (Victoria), PO Box 41. East Melbourne. Victoria 3002. Australia. 